\section{Imaging in Stem Cell Transplant and Cell-Based Therapy}

T. Pandey, Ed.

Cham, Switzerland: Humana Press, 2017, 174 pages, $\$ 179.99$

Regenerative medicine is an approach that can recover normal function of human cells, tissues, or organs injured by trauma or damaged by disease or inflammation. Stem cell therapy, because of its potency for differentiation into specialized cells, is a major biomedical technique in regenerative medicine. Stem cell-based therapy, after rapid advance during the last decades, is moving from the basic science into the clinical care. The role of diagnostic and interventional radiologists and physicians involved in molecular imaging is becoming increasingly important in this bench-to-bedside development, because there are many technical issues in imaging, tracking, and monitoring of stem cells and in the assessment of engraftment efficiency, as well as targeted delivery of stem cells. So far, many scientific articles and reviews have been published introducing stem cell therapy and regenerative medicine. However, a wider explanation of this field is still needed, from the imaging specialist's point of view. Therefore, it is encouraging to see this book providing essential information about stem cell- and cell-based therapies, with a specialized focus on imaging for the radiologists and physicians interested in engagement in this promising field.

This book is organized into 2 parts: "Imaging in Stem Cell Transplant," with 4 chapters, and "Applications in Stem Cell and Cell Based Therapies," with 6 chapters. The first chapter is an overview of molecular imaging techniques in stem cell transplantation. Here, a brief explanation about the potency of stem cells and clinical applications is presented. In addition, various methods of stem cell imaging including MRI, optical imaging, and radionuclide imaging are introduced and compared with each other. The second chapter provides potential applications of nanotechnologies in stem cell research, such as superparamagnetic iron oxide for MRI; gold nanoparticles for optical imaging; and quantum dots, polymer nanoparticles, and silica nanoparticles for fluorescent imaging. The third chapter is about radiologic procedures used in pediatric stem cell transplantation, being specially focused on the role of imaging modalities for assessment of complications after hematopoietic stem cell transplant. Detailed descriptions of imaging findings from various early and later complications and the example CT or MRI scans will attract the interest of imaging specialists. The specialized role of radiolabeled metaiodobenzylguanidine (MIBG) imaging in pediatric patients with neuroblastoma is also introduced in this chapter. The last chapter of the first part introduces clinical applications of stem cell transplant in treating nonhematologic conditions, especially about the unique attributes of mesenchymal stem cells, in various diseases occurring in different systems including the central nervous, musculoskeletal, gastrointestinal, pulmonary, and cardiovascular as well as immunologic and inborn errors of metabolism. The conceptual pictures explaining the role of stem cells are also presented.

COPYRIGHT @ 2018 by the Society of Nuclear Medicine and Molecular Imaging.
The second part, about applications of stem cell therapy, selectively focuses on various clinical fields. Multiple myeloma (MM) for hematologic disease and cardiac regeneration for nonhematologic disease are selected here. The applications of stem cell therapy and imaging in $\mathrm{MM}$ are addressed in chapters 5 and 6 . The standard strategy of the combination of high-dose chemotherapy and autologous stem cell transplantation is outlined in various clinical situations such as those for newly diagnosed, relapsed, or elderly patients with MM. Alternative strategies, including the total therapy concept and the allogeneic stem cell transplantation, are also introduced in chapter 5. Simultaneous MRI and ${ }^{18}$ F-FDG PET imaging for the assessment of tumor burden in a preclinical MM study is compared with other methods including caliper measurement, enzyme-linked immunosorbent assay, and bioluminescence imaging in chapter 6. Stem cell therapy and imaging in cardiac regeneration are presented in chapters 7-9. The seventh chapter focuses on the role of cardiac stem cells (CSCs) in cardiac regeneration. This chapter addresses the phenotype markers of different populations of CSCs, such as c-kit, and the discordant results regarding the role of CSCs in cardiomyocyte renewal. Many preclinical studies and clinical trials with CSCs, as well as ex vivo studies for engineering or stimulation of CSCs, are summarized in detail. Furthermore, other stem cell sources including hematopoietic stem cells, mesenchymal stem cells, and endothelial progenitor cells for heart regeneration are introduced. In the next chapter, various cardiac imaging methods are summarized not only for tracking of transplanted stem cells but also for evaluation of the cardiac functional changes after stem cell therapy. Chapter 9 focuses more specifically on bone marrow cell (BMC) therapy and imaging in ischemic heart disease. The meta-analysis using data from 48 randomized controlled trials of BMC therapy among nearly 80 randomized controlled trials of BMC therapy are summarized. Additionally, the advantages and disadvantages of clinical imaging modalities are discussed. The last chapter of this book refers to a relatively new technique, uterine stem cells, and the future therapeutic potential for clinical applications in regenerative medicine of these cells.

This book has major strengths, including numerous illustrated diagrams regarding imaging materials and modalities as well as treatment strategies and clinical/preclinical images. The detailed summary and discussion of the previous results from basic, preclinical, to clinical fields are sufficient to gain an insight into their potential as well as their current limitations. We recommend this book to clinicians and imaging specialists interested in this increasingly popular field. This book would be helpful as a guide to diagnose unsolved research and clinical problems and when choosing the right method for imaging in stem cell therapy.

June-Key Chung*
Seunggyun Ha
*Seoul National University Hospital
28 Yongon-dong
Chongno-gu, Seoul, 110-744, Korea
E-mail: jkchung@snu.ac.kr

Published online Aug. 16, 2018.

DOI: 10.2967/jnumed.118.219154 